\title{
Mesoscopic Fano effect in an Aharonov-Bohm interferometer Coulomb-coupled to a nearby quantum dot
}

\author{
M. Ţolea ${ }^{* 1}$, V. Moldoveanu ${ }^{1}$, and B. Tanatar ${ }^{2}$ \\ ${ }^{1}$ National Institute of Materials Physics, P.O. Box MG-7, Bucharest-Magurele, Romania \\ ${ }^{2}$ Department of Physics, Bilkent University, Bilkent, 06800 Ankara, Turkey
}

Received 30 July 2006, accepted 18 August 2006

Published online 7 February 2007

PACS 72.25Dc, 72.25.Rb, 73.23.Ra, 73.43.Cd

\begin{abstract}
Motivated by the pionieering experiments of Buks et al. [Nature 391, 871 (1998)] we investigate the visibility of the Fano effect in a single-dot Aharonov-Bohm interferometer which is Coulomb-coupled to a nearby quantum dot. The latter acts as a 'Which Path Detector' and is coupled to two leads on which a finite bias is applied. Using the non-equilibrium Keldysh-Green function formalism we compute the currents through the detector and the interferometer. We take into account the first two contributions to the interaction selfenergy and emphasize the correction to the Landauer formula which appears beyond the single-particle approximation. Particular attention is given to the coherence properties of the interferometer in the presence of the electron-electron interaction between the embedded dot and the detector. We show that when the detector is subjected to a finite bias the amplitude of Aharonov-Bohm oscillations of the current through the interferometer decreases. The Fano line is in turn rather stable under interaction. Our results generalize an earlier work of Silva and Levit [Phys. Rev. B 63, 201309 (2001)] and complement the existing description of the controlled dephasing.
\end{abstract}

(C) 2007 WILEY-VCH Verlag GmbH \& Co. KGaA, Weinheim

1 Introduction and model The electron-electron interaction lies at the origin of some outstanding effects in mesoscopic transport: the Coulomb blockade, the Kondo effect or the Coulomb drag. On the other hand it is generally accepted that the quantum interference is scrambled by the Coulomb repulsion. Given the new challenge of implementing quantum compution algorithms in solid state devices it has become necessary to understand the interplay between coherence and interaction in Coulomb-coupled systems.

In this paper we study the Aharonov-Bohm and Fano effects in a single-dot interferometer with a nearby 'Which Path Detector' (WPD). This system was studied experimentally by Buks et al. [1]. It was found in this setup that due to the mutual Coulomb interaction between the embedded dot and the nearby quantum point constriction (QPC) the current through the detector feels the passage of electrons via the dot. The current measurements also showed that the visibility of the $\mathrm{AB}$ oscillations is reduced when the detector is subjected to a finite bias. Since in the experiment one can tune the transport properties of the detector the suppresion of quantum interference is viewed as a controlled dephasing phenomena. A theoretical approach to the experimental results in Ref. [1] was given by Aleiner et al. [2]. The formalism described the dephasing rate of a quantum dot coupled to leads and interacting to a quantum point constriction. The ring-dot geometry was not considered. A similar problem was also treated by Levinson [3].

The natural framework for computing non-equilibrum properties of interacting systems is the Keldysh formalism [4]. We describe by a tight-binding Hamiltonian a simple interferometer composed of three sites, one of them simulating the quantum dot. Two leads $\alpha, \beta$ are attached to the sites 1 and 3. Their chemical potentials satisfy $\mu_{\alpha}-\mu_{\beta}=e V_{0}, V_{0}$ being the bias applied on the leads. The role of the 'Which

* Corresponding author: e-mail: tzolea@infim.ro 
Path Detector' is played by a single site coupled also to two leads $\gamma, \delta$. Although this is not a quantum point constriction as in the experiments of Buks et al. it turns out that the dephasing process can be captured even in such a simplified model system. The explicit form of the Hamiltonian is as follows:

$$
\begin{aligned}
H & =\sum_{i=1}^{4}\left(\varepsilon_{i}+\delta_{i 2} V_{g}\right) d_{i}^{\dagger} d_{i}+\sum_{i \neq j, i, j=1}^{3} e^{i \varphi_{i j}} t_{i j} d_{i}^{\dagger} d_{j}+U d_{2}^{\dagger} d_{2} d_{4}^{\dagger} d_{4} \\
& +t_{L I}\left(d_{1}^{\dagger} c_{0 \alpha}+d_{3}^{\dagger} c_{0 \beta}\right)+t_{L D}\left(d_{4}^{\dagger} c_{0 \gamma}+d_{4}^{\dagger} c_{0 \delta}\right)+\text { h.c. }
\end{aligned}
$$

$d_{i}, d_{i}^{\dagger}$ are annihilation/creation operators in the interferometer $(i=1,2,3)$ and detector $(i=4)$. Similarly we have on leads the pair $c, c^{\dagger}\left(0 \alpha\right.$ is the lead site coupled to the ring). In Eq. (1) $V_{g}$ simulates the gate voltage applied on the dot (i.e on the second site) and the magnetic flux $\phi$ piercing the ring is included in the Peierls phases $\phi_{i j}$ attached the hopping constants $t_{i j}$. It is expressed in quantum flux units $\phi_{0}$ and specifically we have $\varphi_{12}=\varphi_{23}=\varphi_{31}=2 \pi \phi / 3 \Phi_{0}$ and $\varphi_{j i}=\varphi_{j i}^{*} . U$ is the Coulomb interaction strength between the embedded dot and the detector. $t_{L I}$ and $t_{L D}$ are hopping constants between the lead and interferometer and between lead and detector.

The non-equilibrium Green function is computed from the coupled Dyson equations:

$$
G=G_{\mathrm{eff}}+G_{\mathrm{eff}} \Sigma_{i} G, G_{\mathrm{eff}}=G_{0}+G_{0} \Sigma_{l} G_{\mathrm{eff}},
$$

where $\Sigma_{i}$ is the self-energy due to interactions and the effective Green function describes the noninteracting system in the presence of the leads. While the leads' self-energy $\Sigma_{l}$ is known exactly the interaction selfenergy will be computed perturbatively up to the second order in the interaction strength $U$, i.e we put $\Sigma_{i}=\Sigma_{i}^{1}+\Sigma_{i}^{2}$. The two contributions are expressed in terms of the noninteracting effective KeldyshGreen functions and can be readily computed. Following the standard procedures [5] we obtain a closed formula for the current through the lead $\alpha$ attached to the interferometer (we omit the energy dependence in the integrand for brevity):

$$
\left\langle J_{\alpha}\right\rangle=\frac{e t_{L I}^{2}}{\hbar} \int_{-2 t_{L}}^{2 t_{L}} d E\left(2 \pi \rho^{2} G_{13}^{R} G_{31}^{A}\left(f^{\alpha}-f^{\beta}\right)-\rho G_{12}^{R} \operatorname{Im}\left(2 \Sigma_{i, 22}^{R, 2} f^{\alpha}+\Sigma_{i, 22}^{<, 2}\right) G_{21}^{A}\right),
$$

where $f^{\alpha}, f^{\beta}$ are the Fermi functions of the leads $\alpha, \beta$ and $\rho$ is the lead's density of states. The second term in the current is due entirely to the electron-electron interaction and emphasizes the known fact that the Landauer picture fails beyond the mean-field approach for Coulomb interaction. We shall denote this contribution by $J_{2}$ and the first one by $J_{1}$. In the noninteracting case $J_{1}$ is just the Landauer-like current.

Our calculations are similar to those of Silva and Levit [5] but brings more generality since we have considered explicitly the ring-dot geometry and the correction to the Landauer formula. To our best knowledge, this is the first study that treats that mesoscopic Fano effect in AB intereferometers interacting with a second system.

2 Results In the following we give the main results of our analysis. We have used Eq. (3) to compute the current through the interferometer for various parameters. The interferometer is subjected to a small bias $V_{0}=0.1$ while the bias applied to the leads $\gamma, \delta$ of the detector is allowed to vary. We choose weak ring-dot couplings $t_{12}=t_{23}=0.2$. Fig. 1a shows two Fano lines in the presence of electron-electron interaction between the embedded dot and the detector $(U=0.5$ and $U=0.9)$ at fixed bias $V=0.5$. The two lines are very similar except that the curve corresponding to $U=0.9$ is shifted due to a larger real part of the interacting self-energy. The Fano line is therefore stable against the Coulomb repulsion between the dot and the environment. We have also checked that the current through the detector exhibits a step-like behavior as a function of the gate potential that is applied only to the embedded dot. Therefore the detector is sensitive to resonant processes in the interferometer. 

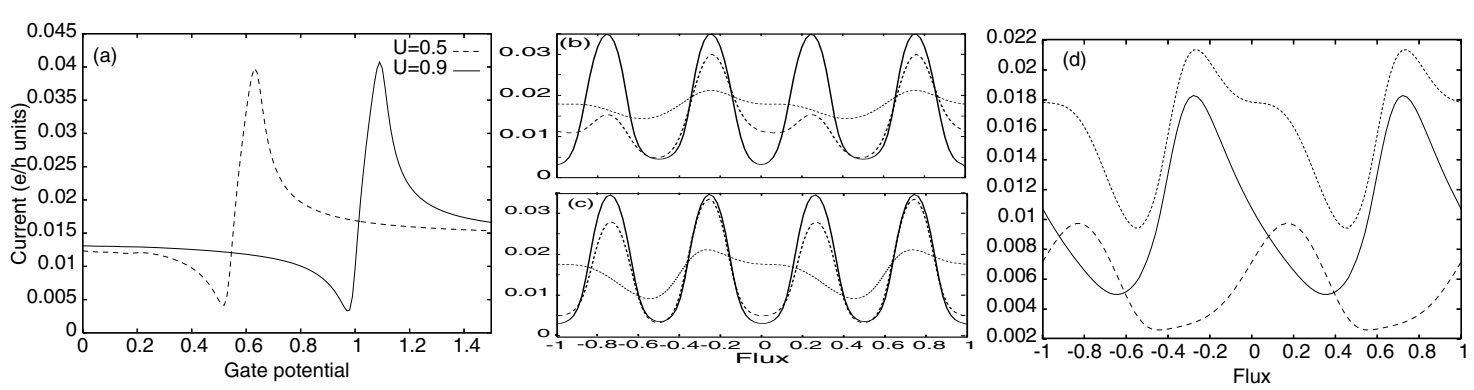

Fig. 1 a) Coulomb-Fano lines at $\phi=0, V=0.5$; full line - $U=0.9$, dashed line - $U=0.5$. b), c) Aharonov-Bohm oscillations at $U=0.5$ (b) and $U=1.0$ (c) for three values of the bias on the detector; full line - $V=0$, dashed line - $V=0.5$, dotted line $V=1.0$. d) The currents $J_{1}$ - full line, $J_{2}$ - dashed line and the total current - dotted line. $U=1.0, V=1.0$.

In Figs. $1 \mathrm{~b}$ and 1c one can see the dephasing effect on the Aharonov-Bohm oscillations (ABO) at finite bias $V$ on the detector. The plots are made at two gate voltages corresponding to the Fano dips of the two lineshapes in Fig. 1a ( $V_{g}=0.52$ for $U=0.5$ and $V_{g}=0.975$ for $\left.U=0.9\right)$. The full lines are clear and periodic ABOs that appear even in the interacting case at $V=0$. This happens because in the absence of bias on the detector the latter does not feel the tunelling processes in the embedded dot. At a formal level one argues that the imaginary part of the interaction self-energy vanishes. The situation changes drastically as $V$ increases. As seen from the dashed and dotted curves in Figs. $1 \mathrm{~b}$ and $1 \mathrm{c}$ the ocillation amplitude decreases and the flux periodicity is lost.

The oscillations are destroyed because the amplitude of their maxima decreases while their minima increases at finite bias. Figure 1d provides further details of the total current at $U=0.9$ and $V=1.0$. The full lines represents the current $J_{1}$ which gives the main contribution. The dashed line shows the correction to the LB formula $J_{2}$. Two features are noticed: i) neither $J_{1}$ nor $J_{2}$ are flux periodic, ii) in several flux ranges $J_{2}$ is comparable to $J_{1}$ or even bigger. Therefore, $J_{2}$ contributes nontrivially to the total current and to the dephasing processes and cannot be neglected. At $V=0$ this correction dissapears (not shown). We have checked that the reduction of the Aharonov-Bohm oscillations is present for other values of the gate potential (e.g at the middle of the Fano line or at the Fano peak) and for other values of the ring-dot coupling. The most damaged oscillations are however the ones around the Fano dip because only in this region the correction $J_{2}$ takes significant values. The current $J_{1}$ near the Fano peak still decreases at higher bias and the associated $\mathrm{AB}$ oscillations are clearer than the ones we presented here.

Summing up, we have shown that the coherence of a single-dot AB interferometer can be destroyed by the Coulomb interaction coming from a quantum dot placed in its vicinity. However, a finite bias is needed on the dot in order to discern the dephasing. Further details about the formalism and a complete analysis of the controlled dephasing are presented elsewhere. [6] Further progress in the understanding of decoherence at nanoscale would involve the inclusion of the intradot interaction as well as the spin-flip effects. The latter were proved to be important in dephasing [7].

Acknowledgements This work was supported by the Research Grant CEEX-2976, TUBITAK and TUBA.

\section{References}

[1] E. Buks, R. Schuster, M. Heiblum, D. Mahalu, and V. Umansky, Nature (London) 391, 871 (1998).

[2] I.L. Aleiner, N.S. Wingreen, and Y. Meir, Phys. Rev. Lett. 79, 3740 (1997).

[3] Y. Levinson, Europhys. Lett. 39, 299 (1997).

[4] Y. Meir and N.S. Wingreen, Phys. Rev. Lett. 68, 2512 (1992).

[5] A. Silva and S. Levit, Phys. Rev. B 63, 201309 (2001).

[6] V. Moldoveanu, M. Ţolea, and B. Tanatar, http://arxiv.org/abs/cond-mat/0604073 (2006).

[7] J. König and Y. Gefen, Phys. Rev. Lett. 86, 3855 (2001). 